\title{
Leukemia vaccine overcomes limitations of checkpoint blockade by evoking clonal T-cell responses in a murine acute myeloid leukemia model
}

\author{
Dina Stroopinsky, ${ }^{1 *}$ Jessica Liegel,${ }^{1 *}$ Manoj Bhasin,${ }^{1 *}$ Giulia Cheloni, ${ }^{1}$ Beena \\ Thomas, ${ }^{1}$ Swati Bhasin, ${ }^{1}$ Ruchit Panchal, ${ }^{1}$ Haider Ghiasuddin, ${ }^{1}$ Maryam \\ Rahimian, ${ }^{1}$ Myrna Nahas, ${ }^{1}$ Shira Orr, ${ }^{1}$ Marzia Capelletti, ${ }^{1}$ Daniela Torres, ${ }^{1}$ \\ Cansu Tacettin, ${ }^{1}$ Matthew Weinstock, ${ }^{1}$ Lina Bisharat, ${ }^{1}$ Adam Morin, ${ }^{1}$ Kathleen \\ M. Mahoney, ${ }^{2}$ Benjamin Ebert, ${ }^{2}$ Richard Stone, ${ }^{2}$ Donald Kufe, ${ }^{2}$ Gordon J. \\ Freeman, ${ }^{2}$ Jacalyn Rosenblatt ${ }^{1 *}$ and David Avigan ${ }^{1 \#}$
} Haematologica 2021 Volume 106(5):1330-1342

${ }^{1}$ Beth Israel Deaconess Medical Center, Harvard Medical School and ${ }^{2}$ Department of Medical Oncology, Dana Farber Cancer Institute, Harvard Medical School, Boston, MA, USA

${ }^{*} D S, J L$ and $M B$ contributed equally as co-first authors.

"JR and DA contributed equally as co-senior authors.

\section{ABSTRACT}

W e have developed a personalized vaccine in which patientderived leukemia cells are fused to autologous dendritic cells, evoking a polyclonal $\mathrm{T}$-cell response against shared antigens and neoantigens. We postulated that the dendritic cell/acute myeloid leukemia fusion vaccine would demonstrate synergy with checkpoint blockade by expanding tumor antigen-specific lymphocytes that would provide a critical substrate for checkpoint blockade-mediated activation. Using an immunocompetent murine leukemia model, we examined the immunological response to and therapeutic efficacy of vaccination in conjunction with checkpoint blockade with respect to leukemia engraftment, disease burden, survival and the induction of tumor-specific immunity. Mice treated with checkpoint blockade alone had rapid progression of leukemia and only a modest extension of survival. Vaccination with dendritic cell/acute myeloid leukemia fusions resulted

\section{Correspondence:}

DINA STROOPINSKY

dstroopi@bidmc.hanard.edu

Received: May 20, 2020.

Accepted: December 11, 2020.

Pre-published: February 4, 2021.

https://doi.org/10.3324/haematol.2020.259457

(C)2021 Ferrata Storti Foundation

Material published in Haematologica is covered by copyright. All rights are reserved to the Ferrata Storti Foundation. Use of published material is allowed under the following terms and conditions:

https://creativecommons.org//icenses/by-nc/4.0/legalcode. Copies of published material are allowed for personal or internal use. Sharing published material for non-commercial purposes is subject to the following conditions:

https://creativecommons.org/licenses/by-nc/4.0/legalcode, sect. 3. Reproducing and sharing published material for commercial purposes is not allowed without permission in writing from the publisher. in the expansion of tumor-specific lymphocytes and disease eradication in a subset of animals, while the combination of vaccination and checkpoint blockade induced a fully protective tumor-specific immune response in all treated animals. Vaccination followed by checkpoint blockade resulted in upregulation of genes regulating activation and proliferation in memory and effector $\mathrm{T}$ cells. Long-term survivors exhibited increased T-cell clonal diversity and were resistant to subsequent tumor challenge. The combination of dendritic cell/acute myeloid leukemia fusion vaccine and checkpoint blockade treatment offers unique synergy, inducing durable activation of leukemia-specific immunity, protection from lethal tumor challenge and the selective expansion of tumor-reactive clones.

\section{Introduction}

While acute myeloid leukemia (AML) is often initially sensitive to cytotoxic therapy, responses are typically transient because of the presence of clonal populations with intrinsic resistance to chemotherapy. ${ }^{1}$ The potency of cell-based immunotherapy for patients with AML is supported by the observation that allogeneic transplantation is uniquely curative for a subset of patients, being mediated by alloreactive lymphocytes. ${ }^{2}$ However, therapeutic efficacy is limited by associated toxicity of graftversus-host disease arising from the lack of specificity of the immune response. ${ }^{3} \mathrm{~A}$ major area of investigation is the development of immunotherapeutic strategies to 
induce disease regression more selectively while providing durable protection from relapse through the establishment of memory responses.

We have developed a personalized tumor vaccine in which patient-derived tumor cells are fused with autologous dendritic cells (DC) such that a broad array of tumorderived antigens, including neo-antigens, is presented in the context of DC-mediated co-stimulation, thereby effectively capturing tumor heterogeneity., In a phase I/II clinical trial, vaccination of AML patients who achieved chemotherapyinduced remission induced durable expansion of leukemiaspecific $\mathrm{T}$ cells in the peripheral blood and bone marrow. Remarkably, despite the vaccinated patients having a median age of 63 years, $71 \%$ of them remained in remission at a median of 5 years of follow-up. ${ }^{6}$ These results were in stark contrast to historical data suggesting a 3-year progression free survival of $10-15 \%$ in this age group.?

A potential challenge to therapeutic efficacy of active vaccination is dysfunction of the T-cell repertoire, characterized by upregulation of pathways that promote exhaustion and senescence, particularly in the microenvironment of advanced disease. ${ }^{8} \mathrm{~A}$ transformative advance in the field of immunotherapy was the finding that therapeutic blockade of the programmed death 1 (PD-1)/programmed death ligand 1 (PD-L1)-negative co-stimulatory pathway resulted in dramatic disease response in a subset of solid tumors, such as melanoma, characterized by a high mutational burden and the presence of neo-antigens and an associated intrinsic T-cell response. ${ }^{9}$ In contrast, checkpoint blockade has shown minimal therapeutic efficacy in patients with hematologic malignancies such as AML, ${ }^{10}$ potentially because of the relatively low mutational burden and lack of a significant population of tumor-reactive lymphocytes within the tumor microenvironment.

We postulated that a combination of vaccine and checkpoint inhibitor therapy would demonstrate unique synergy in which vaccination would provide functionally competent leukemia-specific T-cell populations while the introduction of checkpoint blockade would enhance the effectiveness and persistence of these cells. Because an exhausted T-cell phenotype can be due to several immunoinhibitory signals working in concert, ${ }^{11}$ we hypothesize that simultaneous checkpoint blockade may be advantageous.

In the present study in an immunocompetent murine model of aggressive leukemia, we interrogated the immunological response to and therapeutic efficacy of fusion cell vaccination in conjunction with blockade of negative co-stimulatory pathways using antibodies targeting PD-1 and T-cell immunoglobulin and mucin domain-containing protein 3 (TIM3), critical mediators of the immune suppressive milieu of the bone marrow. ${ }^{12-14}$ We also targeted repulsive guidance molecule $b$ (RGMb), a co-receptor for bone morphogenetic proteins which play a role in the maintenance of hematopoietic progenitors including support for AML cells in the marrow niche $e^{15,16}$ and may also mediate immune tolerance via binding to PD-L2 on myeloid cells. ${ }^{17}$

We demonstrated that the combination of a DC/AML vaccine and checkpoint blockade was uniquely effective in preventing disease progression and inducing a memory response as manifested by protection from tumor re-challenge. Vaccination followed by checkpoint blockade resulted in upregulation of genes regulating activation and proliferation of memory and effector $T$ cells as well as enhanced T-cell clonal diversity.

\section{Methods}

\section{Cell lines}

The murine AML cell line TIB-49 was purchased from the American Type Culture Collection. For all experiments, cell lines were transduced with luciferase/Mcherry using a lentiviral vector (pCDH-EF-eFFLy-T2A-mCherry). Murine LSK cells were obtained from transgenic C57BL/6 mice expressing mIDH2 (IDH2R140O) and subsequently transduced with Hoxa9-GFP and Meis1a-YFP oncogenes, as previously described. ${ }^{18}$ Further details are provided in the Online Supplementary Methods.

\section{Vaccination with dendritic cell/acute myeloid leukemia fusions and/or treatment with checkpoint inhibitors in vivo}

All animal studies were approved by the institutional animal care and use committee of Beth Israel Deaconess Medical Center. Murine syngeneic DC/AML fusion cells were generated as previously described. ${ }^{19} \mathrm{C} 57 \mathrm{BL} / 6$ mice were inoculated retro-orbitally with $5 \times 10^{4}$ luciferase/mCherry TIB-49 murine leukemia cells $(\operatorname{lmTIB})$ or $20 \times 10^{4}$ mutant IDH2/ Hoxa9-GFP/Meis1a-YFP primary AML cells using tail vein injections. Cohorts of mice were assigned to treatment with $100 \times 10^{3} \mathrm{DC} / \mathrm{AML}$ fusion cells via subcutaneous injection $24 \mathrm{~h}$ after AML challenge; intraperitoneally with $200 \mu \mathrm{g}$ each of rat anti-mouse PD-1 (29F.1A10) rat IgG2a,k (BioXCell, NH, USA); mouse anti-mouse TIM3 (T3A.1A10) mIgG1,k; rat antimouse RGMb (307.9D1) rat IgG2a,k or all three monoclonal antibodies starting 4 days after AML challenge and continued every 3 days for six doses; or the combination of DC/AML fusion vaccine and monoclonal antibody treatment. Further details are provided in the Online Supplementary Methods.

\section{Assessment of leukemia-specific immunity}

On day 14-17 following tumor challenge, peripheral blood or spleen-derived $\mathrm{T}$ cells were isolated from the treated animals and exposed to either syngeneic TIB-49 tumor lysate or primary mutant IDH2 tumor lysate for 3 days. Intracellular interferongamma (IFN- $\gamma$ ) expression was then evaluated by intracellular flow cytometric analysis as a measure of leukemia-specific recognition.

To assess antigen-specific anti-tumor immunity the splenocytes underwent flow cytometric analysis using $\mathrm{H}-2 \mathrm{Db}$ pentamers. Cells were stained with anti-CD8APC-Cy7 and murine survivispecific APC-conjugated pentamers ATFKNWPFL (ProImmune, Inc; Sarasota, FL, USA). Cytomegalovirus-specific PE-conjugated pentamers HGIRNASFI (Prolmmune, Inc; Sarasota, FL, USA) were used as the control. The percentage of pentamer-positive CD8 T cells was assessed using multichannel flow cytometry.

\section{Analysis of T-cell receptor diversity}

Targeted T-cell receptor (TCR) diversity was interrogated using a SMARTer human $\alpha / \beta$ profiling kit (Takara, CA, USA). Initially total RNA was extracted and purified from mouse blood using an RNeasy mini kit (Qiagen, Germantown, MD).

The quality of the sequencing data was checked in order to remove low-quality reads and then the data were aligned against TCR sequences from the GenBank and IMGT databases ${ }^{20}$ using MiXCR software. ${ }^{21}$ Further details are provided in the Online Supplementary Methods.

\section{Single-cell RNA sequencing}

Single-cell RNA-sequencing was performed on peripheral blood mononuclear cells isolated from control or treated mice. The 10X Genomics Chromium Controller system ${ }^{21}$ was employed to capture single cells in the context of uniquely barcoded primer beads together in tiny droplets enabling large-scale parallel single-cell 
transcriptome studies. Further details are provided in the Online Supplementary Methods.

\section{Analysis of single-cell RNA sequencing data}

RNA sequencing data were analyzed using standard statistical algorithms after quality control filtering and alignment to the reference genome (mm10) to generate raw counts for transcripts from each cell type. The preprocessed raw count data were log normalized using the Seurat R package (version 3.0) for unsupervised and supervised analyses. ${ }^{22}$ The single-cell RNA sequencing data from samples from the control, vaccine and combination treatment groups were merged using Find Integration Anchors and integration functions in Seurat to generate an integrated matrix of normalized data. ${ }^{23}$ The distribution of various cell-specific markers in a transcriptome profile was determined using the feature plot function in the Seurat R package..$^{24}$ Further details are provided in the Online Supplementary Methods.

\section{Results}

\section{Treatment with checkpoint inhibitors alone is ineffective at preventing disease progression}

The effect of checkpoint inhibition alone on AML engraftment, progression and survival was interrogated in an immunocompetent murine AML model using the TIB49 murine AML cell line. These leukemia cells originated spontaneously in a C57BL/6 mouse and grow aggressively in syngeneic models. As a means of monitoring disease burden, TIB-49 AML cells were genetically manipulated to express luciferase and mCherry (ImTIB-49) via lentiviral transduction and selection. C57BL/6 mice underwent retro-orbital inoculation with $50 \times 10^{3} \mathrm{Im}$ TIB-49 AML cells. The mice were then treated with six doses of isotype control, anti-PD1, anti-TIM3, anti-RGMb or a combination of

A

Day 17

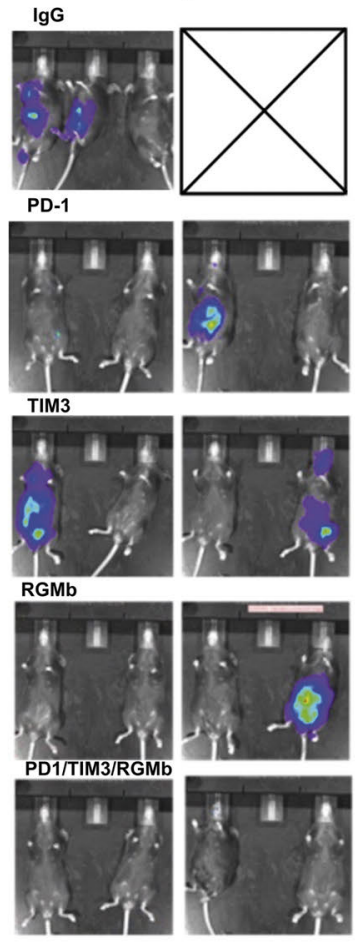

Day 21

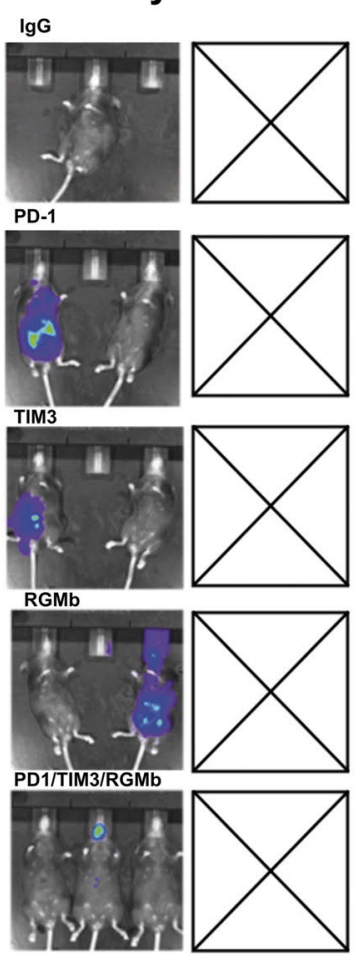

all three monoclonal antibodies starting 3 days after tumor challenge. The treatment doses were administered intraperitoneally every 3 days. Animals were monitored for disease bulk, by serial bioluminescence imaging, and for survival. Untreated animals rapidly developed AML with symptomatic disease requiring euthanasia. Treatment with single-agent anti-PD1, anti-TIM3 or anti-RGMb did not affect AML progression as compared to isotope control, with all animals euthanized by day 53 after tumor challenge whereas the combination of the three monoclonal antibodies modestly delayed the onset of demonstrable leukemia with mildly improved survival (Figure 1A and B).

\section{Checkpoint inhibition in combination with dendritic cell/acute myeloid leukemia fusion vaccine leads to prolonged survival}

We subsequently assessed the effect of checkpoint inhibition in conjunction with DC/AML fusion vaccination. Syngeneic DC/TIB-49 fusion cells were generated as described and confirmed to co-express both DC and tumor markers (Figure 2A). C57BL/6J mice underwent retro-orbital inoculation with $50 \times 10^{3} \operatorname{lm}$ TIB cells. $24 \mathrm{~h}$ after tumor challenge, cohorts of mice were treated with a single dose of $100 \times 10^{3}$ syngeneic fusion cells, 6 doses of anti-PD1/TIM3/RGMb monoclonal antibodies administered IP every 3 days, or the combination of the fusion vaccine and either isotype control or antiPD1/TIM3/RGMb monoclonal antibodies. Serial quantification of disease burden was assessed via bioluminescence imaging analysis beginning approximately 1 month after tumor challenge. All control mice rapidly demonstrated evidence of AML engraftment, progressive disease by day 29 and required euthanasia by day 36 after the initial tumor challenge (Figure 2B). Mice treated with anti$\mathrm{PD} 1 / \mathrm{TIM} 3 / \mathrm{RGMb}$ monoclonal antibodies alone demon-
B

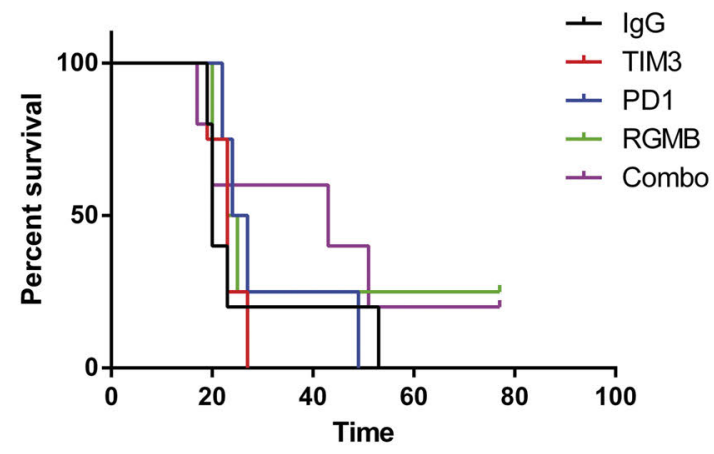

Figure 1. Checkpoint blockade does not significantly affect acute myeloid leukemia engraftment in vivo. C57BL/6J mice were retroorbitally inoculated with $50 \times 10^{3}$ syngeneic TIB-49 acute myeloid leukemia cells that were stably transduced with luciferase/mCherry. The mice were then treated with six doses of $200 \mu$ g anti-PD1, anti-TIM3, or anti-RGMb or a combination (combo) of the three monoclonal antibodies using intraperitoneal injections every 3 days. Some mice were treated with appropriate isotype control as a negative control. To determine the progression of the leukemia, (A) bioluminescence imaging of each group of mice $(n=5)$ was performed on days 17 and 21 after inoculation with the tumor and (B) the mice were followed for survival for 90 days. The results are shown in a Kaplan-Meier curve. 
strated a modest improvement in survival compared to control animals but all required euthanasia by day 44 . The vaccine alone prevented leukemic engraftment in the majority of animals, with two of five mice remaining free of disease at 90 days. Remarkably, the entire cohort of mice treated with vaccination and checkpoint blockade remained alive and disease-free in this model of aggressive AML 90 days after inoculation (Figure 2C).

\section{Checkpoint inhibition in conjunction with a dendritic cell/acute myeloid leukemia fusion vaccine leads to an increase in tumor-specific immunity}

We subsequently examined whether the combination of vaccination with checkpoint inhibition would result in enhanced AML-specific immunity in vivo. To measure tumor-specific T-cell responses, peripheral blood cells were collected from mice 14 days after inoculation and tumor recognition was assessed in a modified enzyme-linked immunospot assay in which the percentage of CD8 T cells exhibiting intracellular IFN- $\gamma$ expression after 3 days of stimulation with autologous TIB-49 tumor lysate was quantified. Mice treated with checkpoint inhibitors alone showed no expansion of $\mathrm{T}$ cells expressing IFN- $\gamma$ following exposure to autologous tumor lysate as compared to control animals which showed mean percentages of $1.9 \%$ for both groups $(n=5)$. Mice treated with the fusion vaccine showed variable expansion of tumor reactive $T$ cells with a mean level of IFN- $\gamma$ expression of $3.4 \%(n=5 ; P=n s)$. This mean expansion did not meet statistical significance because of the variability in tumor-specific $\mathrm{T}$ cells in responding and non-responding animals. However, mice treated with both the fusion vaccine and checkpoint inhibition showed a significant expansion of circulating tumorspecific $\mathrm{CD}^{+} \mathrm{T}$ cells with a mean value of IFN- $\gamma$-expressing cells of $6.4 \%(n=5 ; P=0.01)$ (Figure $3 A$ and $B)$.

In a subsequent experiment, the enhanced expansion of tumor-specific T cells following vaccination and checkpoint inhibition was confirmed in splenocyte populations in animals euthanized 17 days after tumor challenge. Consistent with our prior study, combination therapy resulted in the most pronounced induction of tumor-specific immunity associated with the prevention of leukemia engraftment in all of the treated animals (Figure 3C-E).

To further elucidate the capacity of fusion cell vaccination and checkpoint blockade to elicit tumor-specific immunity in vivo, we quantified antigen-specific $T$ cells targeting survivin following treatment with a checkpoint inhibitor, vaccination or combination therapy. Survivin is a member of the Inhibitor of apoptosis family, known to be overexpressed in AML. Using intracellular flow cytometric analysis, we demonstrated high levels of survivin expression by TIB-49 cells (Figure 3F). Expansion of survivin-specific T

A
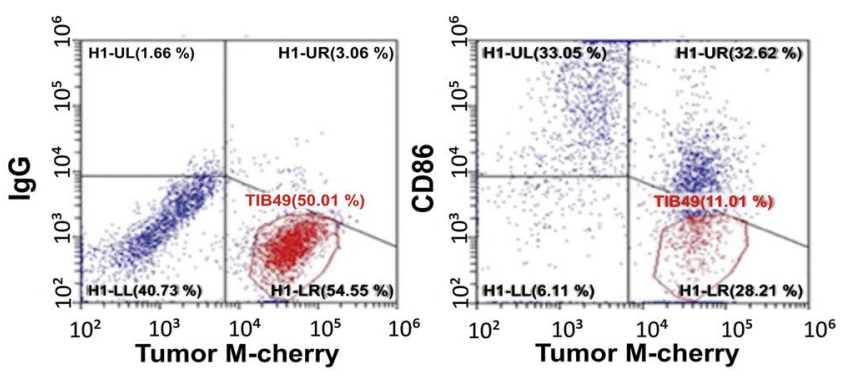

B

Day 29

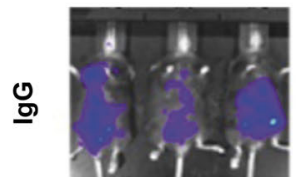

产
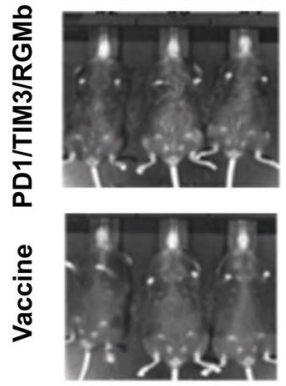

家
Day 36
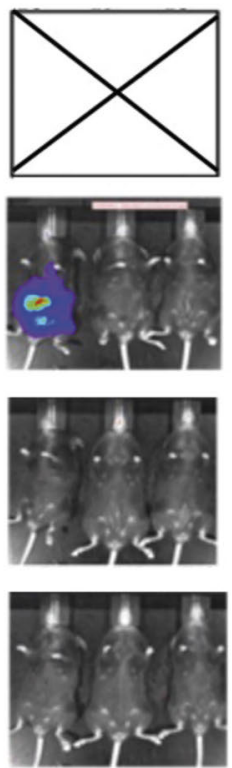

Day 41
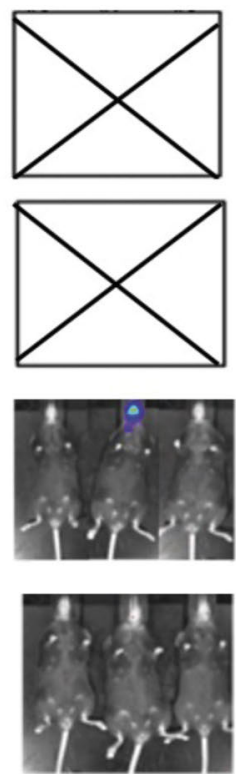

Figure 2. Combination treatment with the fusion vaccine and PD1/TIM3/RGMb blockade prevents establishment of acute myeloid leukemia in vivo. C57BL/6J mice were retro-orbitally inoculated with $50 \times 10^{3}$ syngeneic TIB-49 acute myeloid leukemia (AML) cells that were stably transduced with luciferase/mCherry. (A) Syngeneic dendritic cell (DC)/AML fusion cells were generated as described and evaluated for co-expression of tumor (mCherry) and DC (CD86) markers using flow cytometry. The mice ( $n=5$ in each group) were then treated with either vaccine alone, anti-PD1/TIM3/RGMb (mAbs) or a combination of anti$\mathrm{PD} 1 / \mathrm{TIM} 3 / \mathrm{RGMb}$ and the fusion vaccine. One group of mice was treated with appropriate isotype control as a negative control. (B) Bioluminescence imaging was performed serially starting on day 29 after inoculation (3 representative mice are shown). (C) The mice were followed for survival for 90 days. The results are shown in a Kaplan Meier curve.
Overall survival

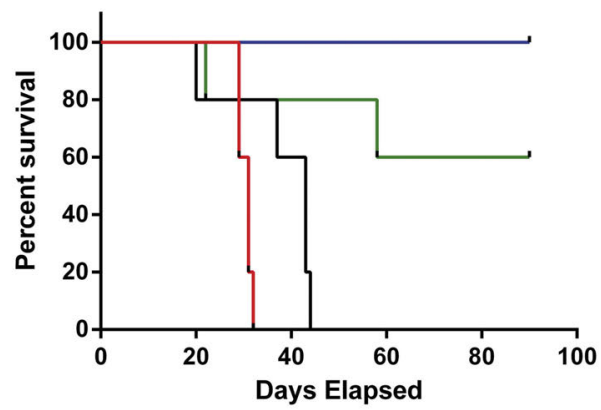


cells was quantified by MHC class I pentamer analysis. Compared to single-agent treatment, vaccination in combination with anti-PD1/TIM3/RGMb monoclonal antibody treatment resulted in a statistically significant 1.8fold expansion of spleen-derived $\mathrm{T}$ cells recognizing murine survivin in spleen $\mathrm{CD}^{+} \mathrm{T}$ cells (Figure $3 \mathrm{G}$ and $\mathrm{H}$ ). Consistent with the tumor specificity of this response, vaccination and checkpoint inhibition did not result in an increased frequency of cytomegalovirus-specific $\mathrm{CD}^{+} \mathrm{T}$ cells.

To assess whether combination therapy with the fusion vaccine and checkpoint inhibition induces a memory response consistent with durable protection, we next interrogated the $\mathrm{T}$-cell repertoire in the peripheral blood from surviving animals at day 36 after AML challenge. Combination treatment with the fusion vaccine and anti$\mathrm{PD} 1 / \mathrm{TIM} 3 / \mathrm{RGMb}$ monoclonal antibodies led to a statisti- cally significant increase in the number of CD4/CD44 $/ \mathrm{CD} 6 \mathrm{~L}^{-}$memory $\mathrm{T}$ cells as compared to the numbers following single-agent treatments. Furthermore, there was a statistically significant decrease in $\mathrm{CD}^{+}{ }^{+} \mathrm{CD} 25^{+} \mathrm{FOXP}^{+}$regulatory $\mathrm{T}$ cells after combination treatment compared to that after vaccination (Online Supplementary Figure S1).

Induction of robust anti-tumor immunity after combinatorial treatment with the syngeneic DC/AML fusion vaccine and checkpoint blockade was reproduced in an immune-competent mutant IDH2 inducible primary AML model. Mice were challenged with 200,000 GFP+ leukemia cells inoculated via the tail vein and then treated with the syngeneic DC/AML fusion vaccine and/or anti$\mathrm{PD} 1 / \mathrm{TIM} 3 / \mathrm{RGMb}$ monoclonal antibodies, as described above. Analysis of spleens harvested from animals treated with the fusion vaccine and checkpoint inhibitors demon-
A

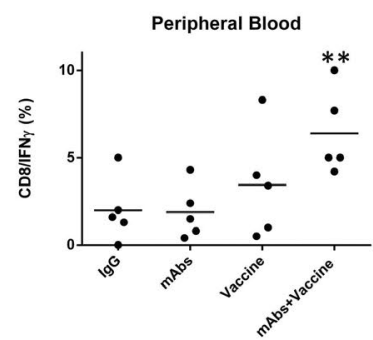

C

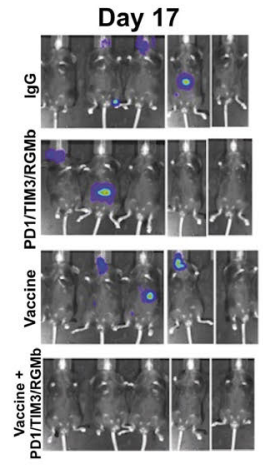

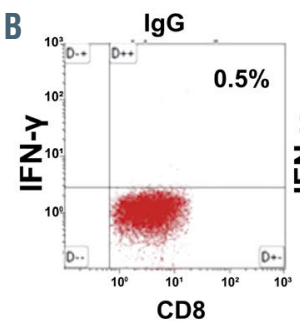
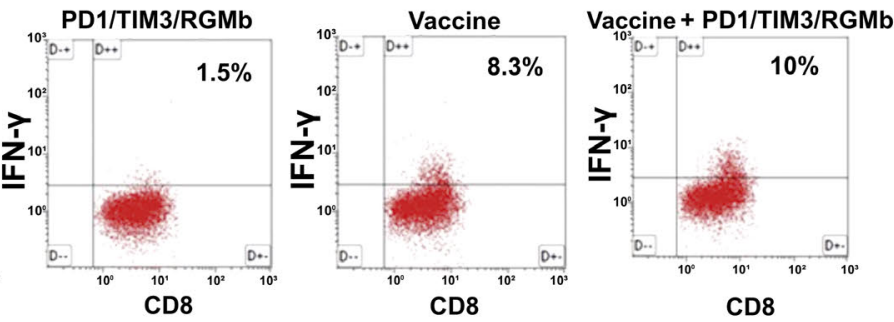

D

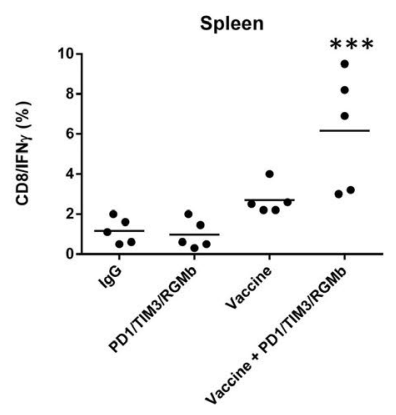

E

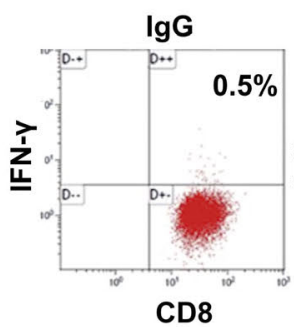

G

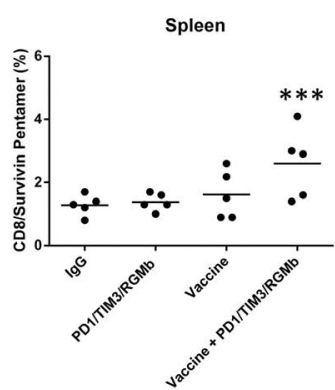

PD1/TIM3/RGMb

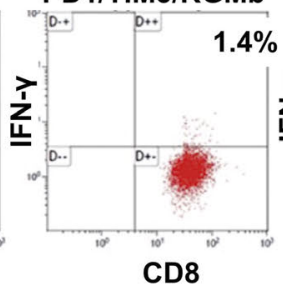

CD8

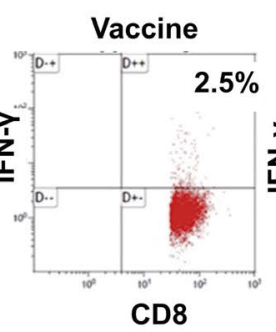

CD8
Vaccine+ PD1/TIM3/RGMb

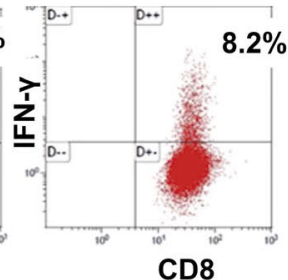

CD8

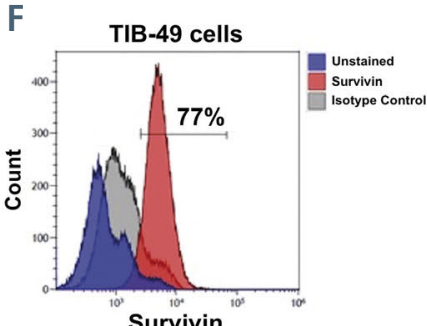


strated significantly decreased leukemia burden compared to that of controls as detected from the GFP signal via flow cytometry (Figure 3I and J).

Peripheral blood $\mathrm{T}$ cells were obtained from animals on day 14 after tumor challenge. Consistent with our previous findings, intracellular flow cytometric analysis demonstrated a 4-fold increase in circulating tumor-specific $\mathrm{CD}^{+} \mathrm{T}$ cells after treatment with the fusion vaccine and checkpoint blockade compared to the numbers in controls detected following ex vivo stimulation with autologous tumor lysate (Figure $3 \mathrm{~K}$ and $\mathrm{L}$ ). Treatment with a dendritic cell/acute myeloid leukemia
fusion vaccine and checkpoint inhibition is protective upon re-challenge with acute myeloid leukemia

Having demonstrated that animals treated with the combination of vaccination and checkpoint blockade develop leukemia-specific immunity with a memory phenotype and durable disease response, we subsequently assessed whether combination vaccine and checkpoint inhibitor therapy provided long-term protection from disease rechallenge.

H
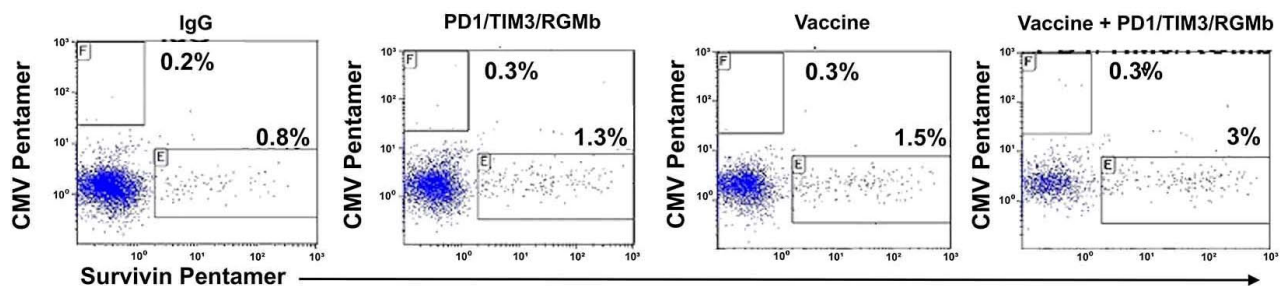

$$
\begin{aligned}
& \text { - Control } \\
& \text { - PD1/TIM3/RGMb } \\
& \text { - Vaccine } \\
& \text { - Vaccine + PD1/TIM3/RGMb }
\end{aligned}
$$

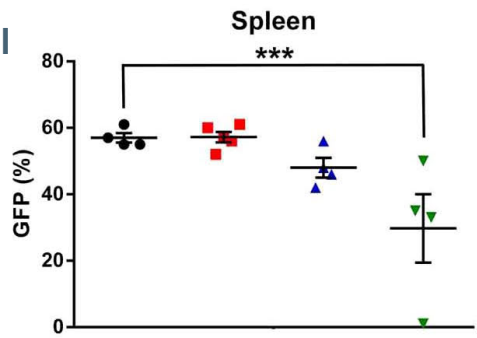

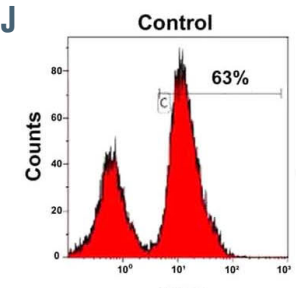

GFP

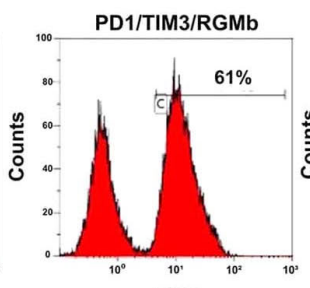

GFP
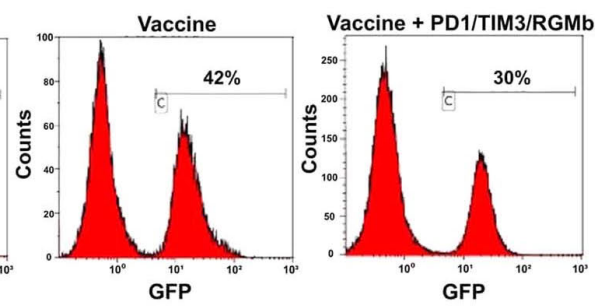

K

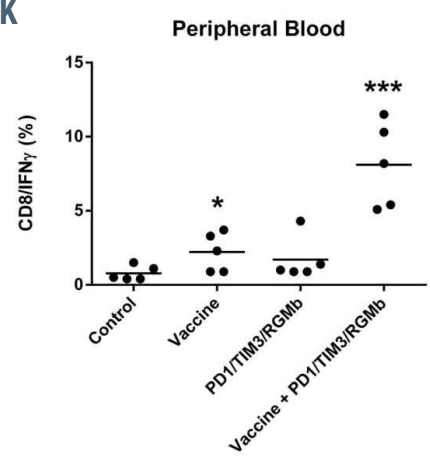

$\mathbf{L}$
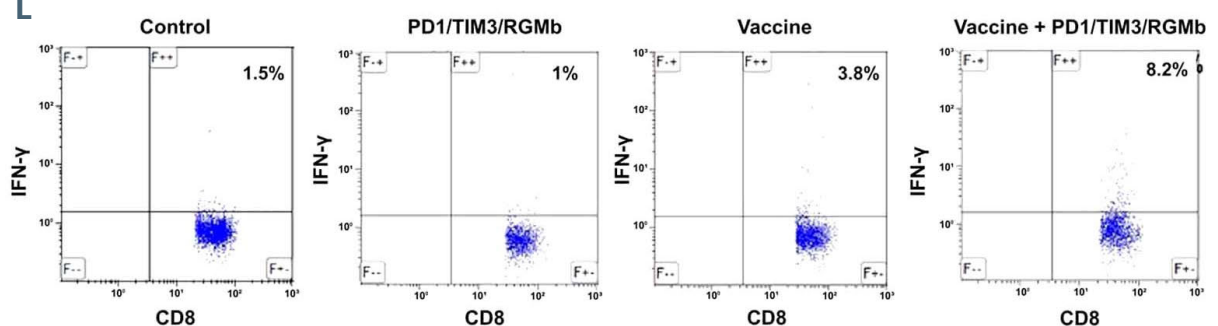

Figure 3. Increase in tumor-specific T cells following combination treatment with the fusion vaccine and PD1/TIM3/RGMb blockade. C57BL/6J mice were treated as described in Figure 2. (A, B) On day 14 peripheral blood was collected and CD8 ${ }^{+} \mathrm{T}$ cells were assessed for intracellular interferon-gamma (IFN $\gamma$ ) expression using multichannel flow cytometry following exposure to autologous tumor lysate for 3 days. Results are presented as a summary of data from the five mice in each group $(* * P<0.05)(A)$ and representative dot plots (B). (C-E) In a similar independent experiment on day 17 after tumor challenge, the mice underwent bioluminescence imaging analysis (C). The mice were then euthanized; splenocytes were harvested and assessed for IFN $\gamma$ expression using multichannel flow cytometry following exposure to autologous tumor lysate. Results are presented as a summary of data from the five mice in each group ( $* * * P<0.05)(D)$ and as representative dot plots (E). (F) Spleen-derived CD8 ${ }^{+} \mathrm{T}$ cells were also assessed for frequency of tumor antigen-specific T cells with multichannel flow cytometry using pentamer analysis. TIB-49 acute myeloid leukemia (AML) cells were confirmed to express survivin using intracellular flow cytometric analysis; unstained cells and cells incubated with an appropriate isotype control were used as negative controls. Binding of APC-labeled multimeric MHC/survivin peptide complexes to T-cell receptors was examined to determine the frequency of the survivin-specific T cells. $(G, H)$ The frequency of survivin-specific $T$ cells is presented in gated CD $8^{+} T$ cells as a summary of data from the five mice in each group $(* * * P<0.05)(G)$ and as representative dot plots $(\mathrm{H})$. The frequency of cytomegalovirus-specific $T$ cells was analyzed as a control. C57BL/6J mice were inoculated by tail vein injection with $200 \times 10^{3}$ primary syngeneic mutant IDH2 AML cells that were stably transduced with green fluorescence protein (GFP). Syngeneic DC/mIDH2 AML fusion cells were generated. The mice were then treated with either vaccine alone, anti-PD1/TIM3/RGMb or a combination of anti$\mathrm{PD1}$ /TIM3/RGMb and the fusion vaccine. Some mice were treated with an appropriate isotype control as a negative control. (I, J) Disease burden was assessed in spleens of animals at day 36 after tumor challenge by detection of the GFP signal via flow cytometric analysis. Results are presented as a summary of data from the five mice in each group $(* * * P<0.05)(\mathrm{I})$ and as representative histograms $(\mathrm{J})$. On day 14 peripheral blood was collected and CD $8^{+} \mathrm{T}$ cells were assessed for intracellular IFN $\gamma$ expression using multichannel flow cytometry following exposure to autologous tumor lysate for three days. Results are presented as (K) a summary of 5 analyzed mice $(n=5 ; P<0.05)$ and $(L)$ as representative dot plots. 
A

Naïve Control

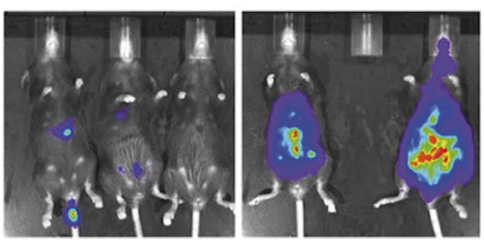

Vaccine + PD1/TIM3/RGMb

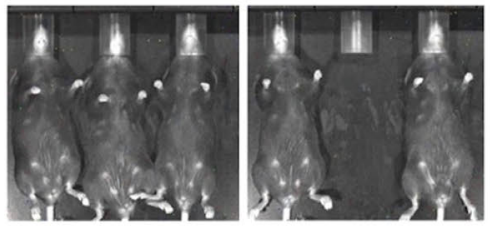

B

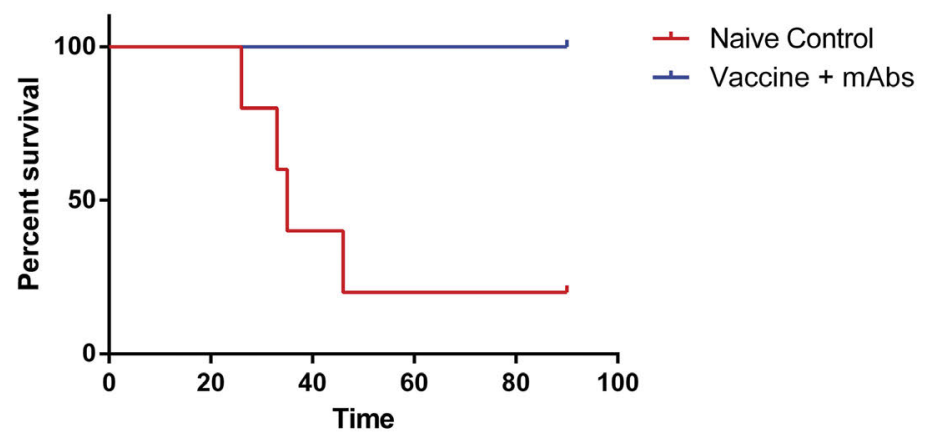

Figure 4. Treatment with the fusion vaccine in combination with PD1/TIM3/RGMb blockade prevents establishment of acute myeloid leukemia upon re-challenge with tumor cells. Ninety days after the initial tumor challenge and treatment with six doses of anti-PD1/TIM3/RGMb (mAbs), C57BL/6J mice ( $n=5)$ were challenged with an additional dose of 50,000 luciferase/mCherry-transduced syngeneic TIB-49 cells. Naïve, age-matched mice ( $n=5)$ were inoculated with the same dose as controls. To assess AML burden, (A) bioluminescence imaging was performed after re-challenge and (B) the mice were followed for survival for 90 days, as illustrated in a Kaplan-Meier curve.

Mice that had initially been challenged with tumor inoculation and rendered disease-free by combined vaccine and checkpoint inhibitor therapy were subsequently re-challenged via retro-orbital inoculation with a lethal dose $\left(5 \times 10^{3}\right)$ of $\operatorname{lmTIB}$ at day 90 . Age-matched naïve C57BL/6J control mice were challenged with $5 \times 10^{3} \mathrm{ImTIB}$ as controls. Mice were followed for survival and disease progression with bioluminescence imaging. In contrast to control animals, mice that had been previously treated with the combination of vaccination and checkpoint inhibition were uniformly protected from disease and showed no evidence of leukemic engraftment (Figure $4 \mathrm{~A}$ and $\mathrm{B}$ ).

We subsequently examined the relative contribution of each individual checkpoint inhibitor on vaccine efficacy. C57BL/6 mice were treated with syngeneic fusion vaccine in combination with anti-PD-1, anti-TIM3 or anti-RGMb monoclonal antibodies, as described above. At day 90 after tumor challenge, three out of five mice treated with the fusion vaccine alone were free of disease, replicating our previous findings. The addition of anti-RGMb did not increase the efficacy shown by that of the fusion vaccine alone, with two out of five animals surviving. However, vaccination in conjunction with PD-1 blockade or TIM3 blockade alone resulted in $100 \%$ of animals surviving without evidence of disease (Figure $5 \mathrm{~A}$ and $\mathrm{B}$ ).

Among the cohorts with complete response to treatment, i.e., those given vaccination and either anti-PD-1 $(n=5)$ or anti-TIM-3 $(n=5)$, mice were re-challenged with $50 \times 10^{3}$ ImTIB cells to evaluate long-term anti-leukemia immunity. Mice were followed for an additional 90 days, after which it was found that all five of the mice in the cohort given the vaccine plus atni-PD-1 remained disease-free after re-challenge, whereas two of five mice in the TIM-3 cohort succumbed to disease (Figure 5C).

\section{Vaccination in conjunction with PD-1 blockade leads to an Increase in inflammatory signaling pathways and blunts apoptosis of memory T cells}

The nature of the immune response was further characterized by interrogation of the immune transcriptome following vaccination in the context of PD-1 blockade as compared to checkpoint inhibition or vaccination alone.
Unsupervised analysis by single-cell RNA sequencing identified comparable numbers of single-cell clusters in all three cohorts of mice (Figure 6A). Supervised analysis of CD4/IL7R and CD8/IL7R memory T cells demonstrated activation/upregulation in signaling pathways regulating $\mathrm{T}$ cell viability, proliferation and survival after vaccination, which were further enhanced after treatment with both the fusion vaccine and checkpoint blockade. Consistent with these findings, there was significant downregulation in apoptosis-regulating genes (Figure 6B). A similar pattern was observed in CD8/Nkg7-expressing effector T cells with increases in viability, activation and proliferation and downregulation of apoptosis genes (Figure 6B).

Furthermore, signaling pathway analysis in the CD8/CDIL7R and CD4/CDIL7R memory compartment demonstrated significant effects on $\mathrm{MTOR}, \mathrm{CD} 28$ and TCR signaling following vaccination compared to untreated controls. Interestingly, these signaling pathways were further upregulated following the addition of checkpoint blockade (Figure 6C and D). Analysis of signaling in effector CD8/Nkg7 cells showed significant upregulation of $\mathrm{NF \kappa B}$, mTOR, CD28 and ICOS signaling after vaccination, consistent with induction of an inflammatory phenotype. Combination treatment with the vaccine and anti-PD-1 further enhanced the activation of these signaling pathways (Figure $6 \mathrm{E}$ ). CD8/Nkg7+ effector T cells also showed a significant impact on TCR signaling pathways $(-\log P$ value $=$ $3.9)$; this effect was further enhanced $(-\log P$ value $=4.7)$ by addition of checkpoint blockade (Figure $6 \mathrm{E})$.

\section{Vaccination in combination with checkpoint blockade results in greater clonal T-cell diversity}

The effect of vaccination alone or in conjunction with checkpoint blockade on the T-cell repertoire was interrogated by clonotypic analysis using targeted TCR profiling studies. The diversity index and rarefaction analysis demonstrated that vaccination resulted in the selective expansion of clonal populations and enhanced diversity of the T-cell repertoire compared to peripheral blood samples derived from untreated control mice (Figure $7 \mathrm{~A}$ and $\mathrm{B}$ ). Remarkably, diversity was significantly further expanded by sequential therapy with DC/AML fusions and combined 
A

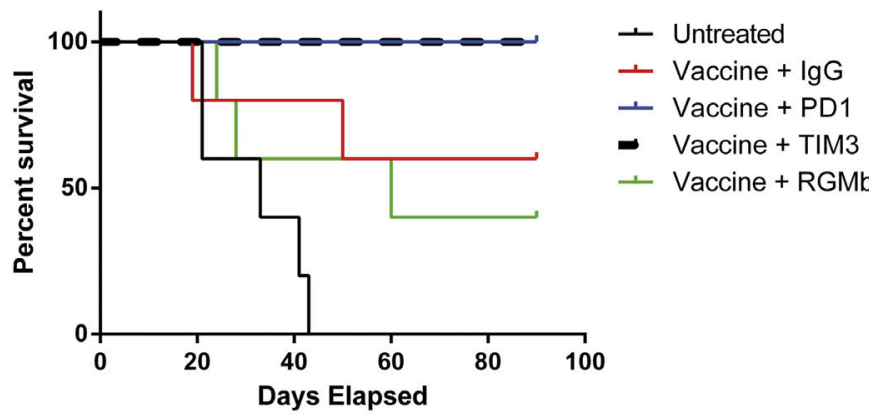

B

Day 30

Day 55

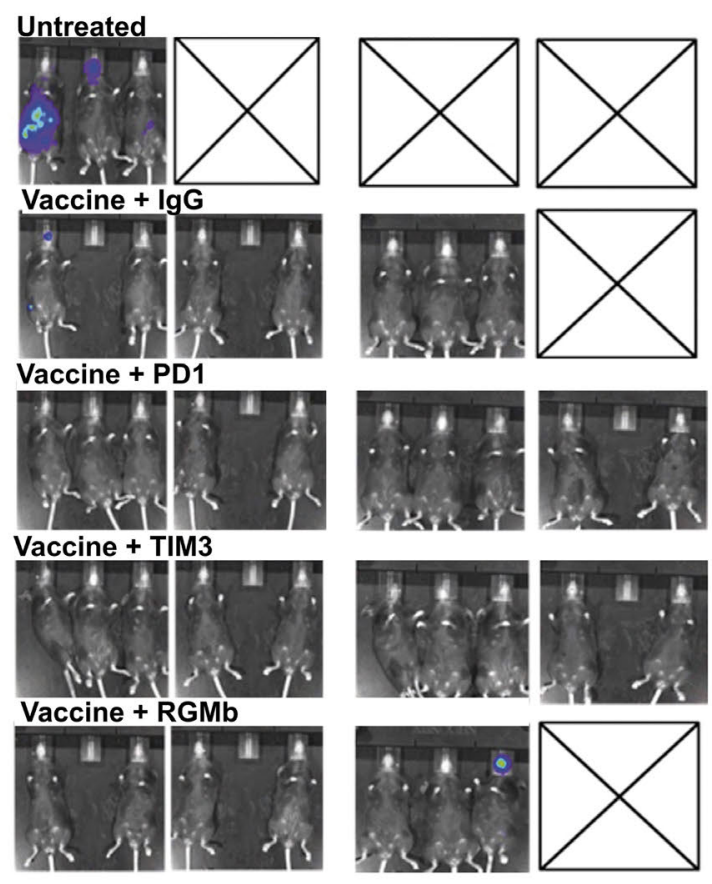

checkpoint blockade, suggesting the expansion of vaccineeducated cells with tumor specificity. Clone tracking analysis of the top TCR clones indicated that multiple TCR clones were significantly modulated (absolute fold-change $>2$ ) after vaccine treatment (Figure 7C). Interestingly, a subset of these vaccine-modulated TCR clones showed further enhanced up- or down-regulation on combined therapy, indicating a synergistic impact of vaccine and checkpoint therapy in building anti-tumor immunity (Figure 7C, Online Supplementary Figure S2). In summary, combination therapy with vaccination and checkpoint blockade resulted in selective, further expansion of vaccine-educated cells creating a pattern of enhanced clonal dominance.

\section{Discussion}

Greater understanding of the mutational landscape in AML has resulted in better prognostication and the development of targeted therapies for subsets of patients. However, while the use of standard chemotherapy and targeted agents has resulted in higher rates of response, a cure remains elusive for the majority of patients. Allogeneic
Figure 5. Combination treatment with the fusion vaccine + anti-PD1 or fusion vaccine + anti-TIM3 prevents the establishment of acute myeloid leukemia in vivo. C57BL/6J mice were retro-orbitally inoculated with $50 \times 10^{3}$ syngeneic TIB-49 acute myeloid leukemia (AML) cells that were stably transduced with luciferase/mCherry. Syngeneic dendritic cell (DC)/AML fusion cells were generated. The mice were then treated with either vaccine alone, or with fusion vaccine in combination with anti-PD1; anti-TIM3 or anti-RGMb. Control mice were treated with the appropriate isotype control. To assess the progression of AML, (A) bioluminescence imaging was performed starting on day 30 after inoculation and (B) the mice were followed for survival as illustrated in the Kaplan Meier curve ( $n=5$ in each group). At 90 days after the initial tumor challenge, mice treated with vaccine + anti-TIM3; and vaccine + anti-PD-1 were re-challenged with an additional dose of $50 \times 10^{3}$ syngeneic TIB- 49 AML cells. (C) The mice were followed for survival for another 90 days. The results are shown in a Kaplan Meier curve.
C

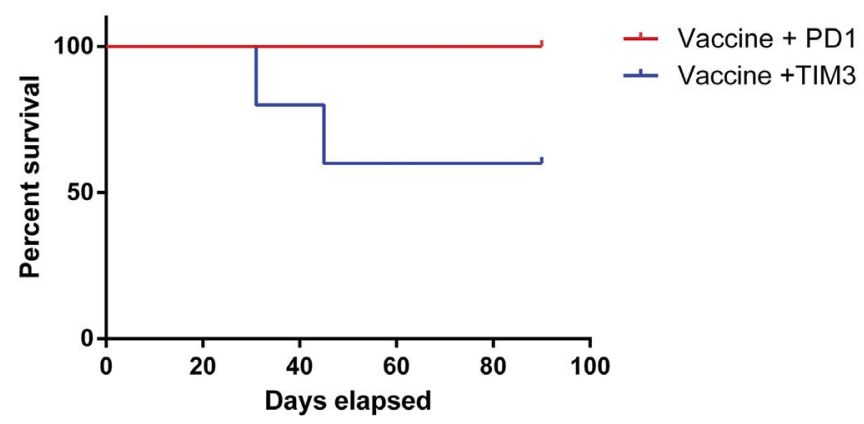

transplantation is curative for a subset of patients because of the graft-versus-disease effect mediated by alloreactive lymphocytes. ${ }^{2}$ This treatment strategy suggested that cellular immune-based therapy was capable of fully eradicating malignant hematopoiesis, including that of the primitive stem cell compartment, albeit with significant associated toxicity due to concurrent risks of graft-versus-host disease and infection. There has been significant interest in developing more targeted immune-based therapies utilizing effector cells from the patient without the need for transplantation.

A major advance in cancer immunotherapy was the discovery of the role of negative co-stimulatory factors such as CTLA-4/B7 and the PD-1/PD-L1 pathways in promoting Tcell exhaustion in patients with malignancy, thereby facilitating immune escape and disease growth. Blockade of these negative checkpoints has led to dramatic disease regression and improved outcomes in a subset of patients with advanced solid tumors, transforming the therapeutic landscape and demonstrating the truly unique potency of immune effector cells. ${ }^{25}$ However, despite the susceptibility to immune-based targeting demonstrated with allogeneic transplantation, the efficacy of checkpoint inhibitors has 

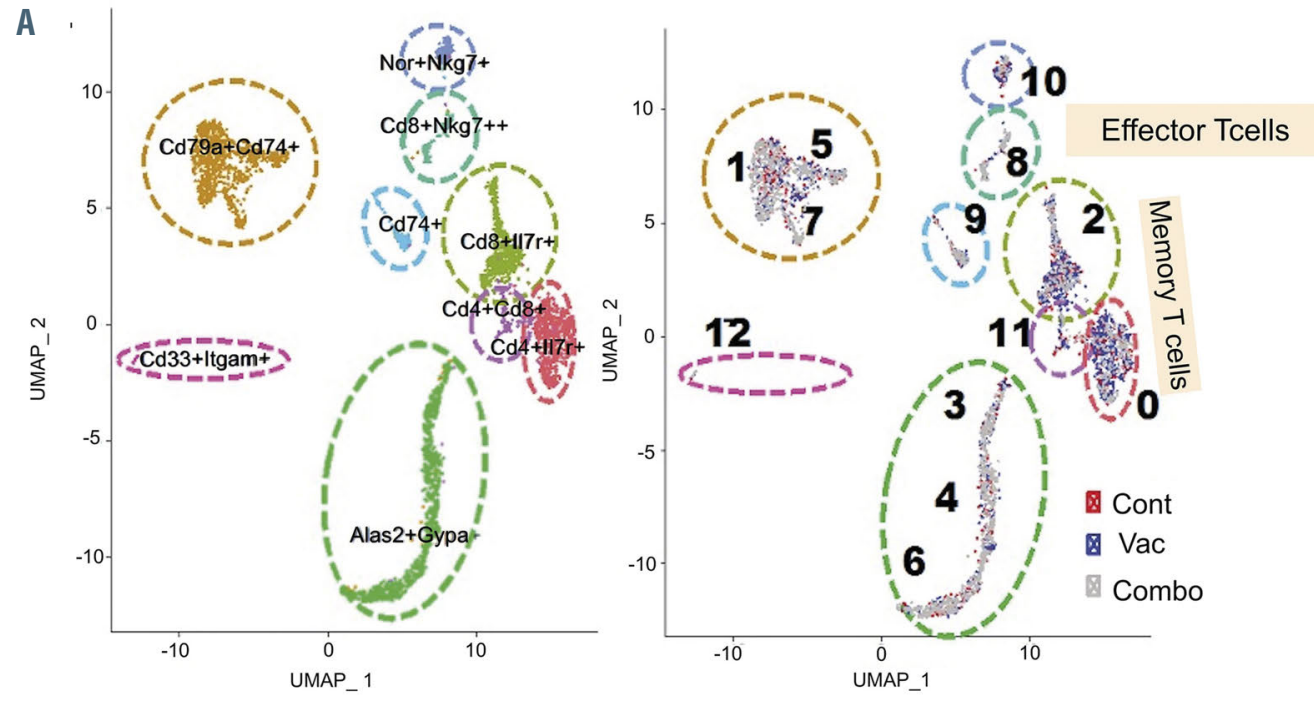

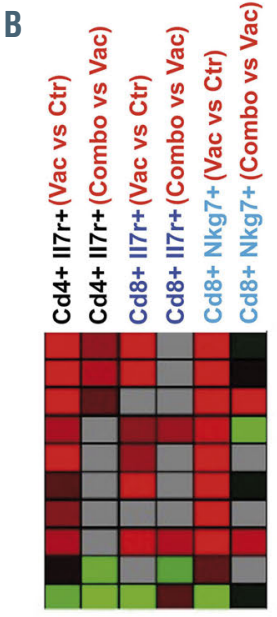

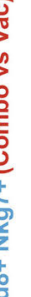

$-2$.

0.0

Cell survival

Cell viability

Activation of cells

T cell development

Proliferation of hematopoietic progenitor cells

Cell viability of lymphocytes

Cell viability of leukocytes

Proliferation of lymphatic system cells

Apoptosis of T lymphocytes

Apoptosis

C

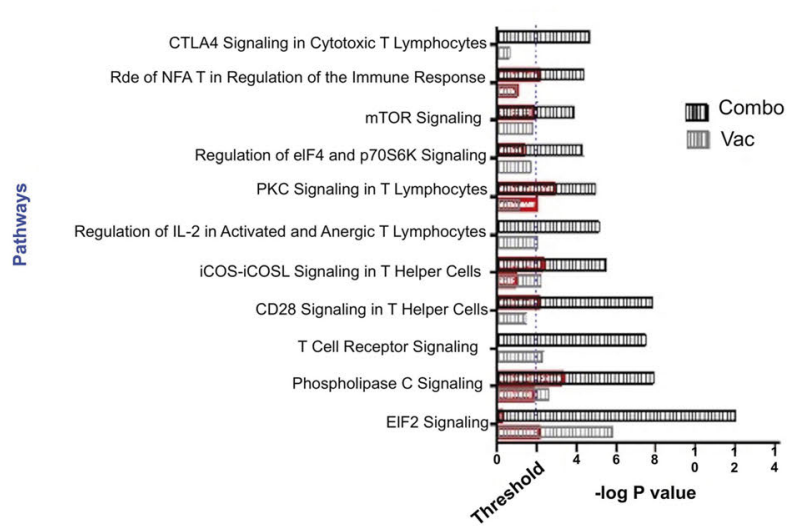

D

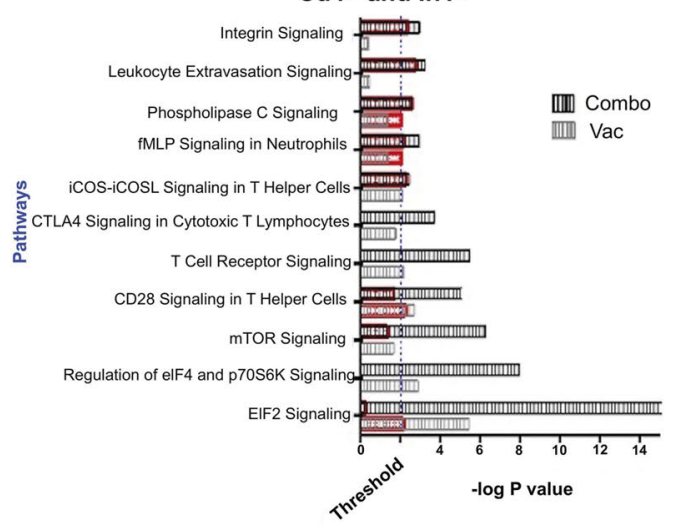

E

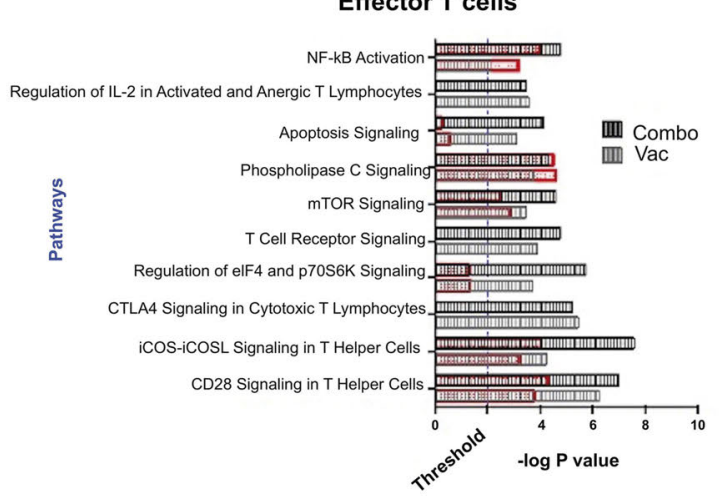

Figure 6. Single-cell RNA sequencing analysis demonstrates that vaccination alone and in combination with checkpoint inhibition affects the T-cell landscape. (A) Singlecell RNA-sequencing analysis of peripheral blood mononuclear cells isolated from control mice, mice treated with the dendritic cell (DC)/acute myeloid leukemia (AML) fusion vaccine, and mice treated with the vaccine + checkpoint point inhibitors. ( $n=3$ mice/group). Visualization of single-cell clusters was achieved using the UMAP approach from normalized data of 710 control, 884 vaccine-treated and 1,489 combination-treated peripheral blood mononuclear cells. Cell clusters were annotated based on expression of established immune-cell markers (e.g., T cells [CD3+], B cells [CD19+], memory T cell [II7r+], and effector cells [Sell', CD62L-] (left panel). Relative proportions of cells in the clusters from each cohort are depicted with different colors (right panel). (B) Functional enrichment heatmap depicting increased (red) or decreased (green) functional categories in the samples from animals treated with vaccine alone or the combination (combo). The heatmap was prepared based on $z-$ scores calculated using ingenuity pathways analysis systems. (C-E) Pathways that are significantly affected in various subsets of memory $T$ cells: CD8a and II7 $\mathrm{r}^{+} \mathrm{T}$ cells (C), CD4 and II7r+ $T$ cells (D), and effector T cells (E). Black and gray bars represent the significance of the impact of vaccine alone and in combination with checkpoint inhibition, respectively, on selected signaling pathways. The extent of activation/increase of various significantly affected pathways is shown using overlapping orange bars. 
A

\section{Diversity Index}
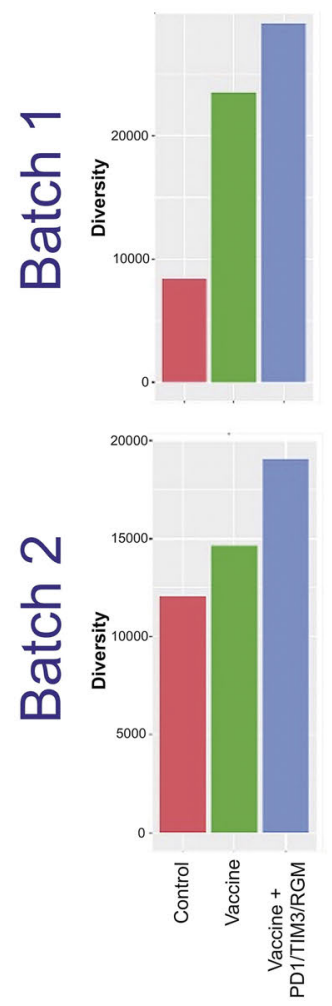

C

\section{Clonality Analysis}

B

\section{Rarefaction}
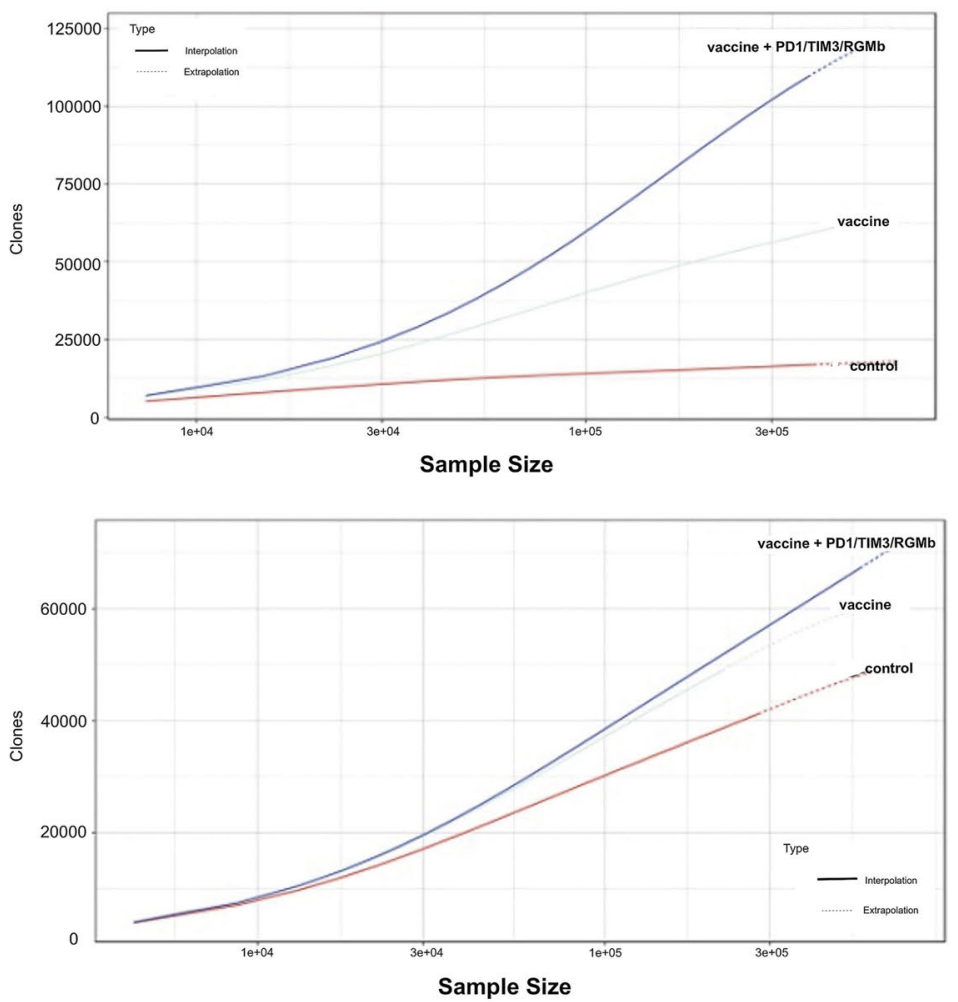

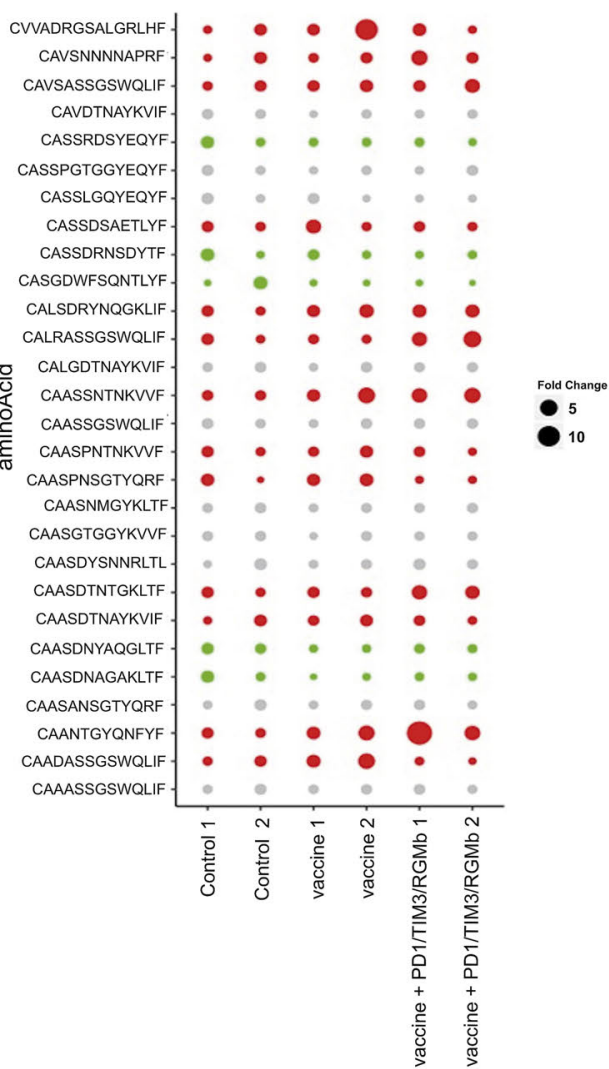

Figure 7. Vaccination with the fusion vaccine leads to greater clonal T-cell diversity, which is further enhanced following checkpoint blockade. C57BL/6J mice were treated as described in Figure 6. Peripheral blood (PB) was then collected and assessed for T-cell diversity using targeted T-cell receptor (TCR) profiling. (A) Inverse Simpson diversity index indicating that vaccine alone and in combination with checkpoint inhibitors enhance T-cell diversity (B) Refraction diversity analysis. (C) Bubble plot of the expression of the top TCR clones after vaccination alone or in combination with checkpoint inhibitors. Columns represent samples and rows represent amino acid sequences of different TCR clones. The TCR clones with significant increases or decreases are represented by red and green, respectively. The fold-change of the top ten TCR clones for each sample is calculated compared to the untreated control samples. 
been disappointing in hematologic malignancies, including AML. ${ }^{26}$ It has been postulated that the lack of a significant intrinsic T-cell response in AML likely explained why there was an insufficient immune substrate for checkpoint inhibition to be effective.

We have developed a personalized tumor vaccine in which patient-derived tumor cells are fused with autologous DC so that a broad array of tumor antigens, including unique neo-antigens, are presented in the context of DCmediated co-stimulation. ${ }^{4,5}$ In a phase II clinical trial, vaccination was associated with a dramatic and durable expansion of leukemia-specific $T$ cells in the peripheral blood and marrow and was associated with more than $70 \%$ of patients remaining in remission with a mean follow-up of 5 years, despite the study patients having a mean age of 63 years. ${ }^{6}$ Of note, we demonstrated that leukemia-specific $T$ cells were nearly absent in the peripheral blood and marrow prior to vaccination. We postulated that DC/tumor fusions would create the necessary expansion of tumor-specific $T$ cells, which could then be further activated and expanded by the presence of checkpoint inhibition. We hypothesized that combined therapy with vaccination and checkpoint inhibition would be synergistic in providing effector cells that maintain a state of activation in the context of the immunosuppressive milieu of the tumor microenvironment.

In the present study, we established an immunocompetent murine AML model in which disease burden could be quantified and tracked by bioluminescence imaging. In this model of aggressive disease, we demonstrated that challenge with syngeneic AML cells resulted in rapid engraftment and death of the host animals within 30 days. Consistent with prior clinical experience the introduction of checkpoint inhibition, via a mix of antibodies targeting PD1 , TIM3, and RGMb, only modestly slowed disease progression and did not significantly affect the survival of the mice. Of note, vaccination with syngeneic DC/AML cells $24 \mathrm{~h}$ after tumor challenge, at a state of minimal disease, markedly slowed disease progression and resulted in longterm survival in a significant subset of animals. As most AML patients with active disease have a deficient immune system, the ideal clinical setting for vaccination is in low tumor burden states, which are more favorable to allow priming of the immune response and induction of longterm memory. This has been demonstrated in subset analyses from several trials of cancer vaccines in which vaccine effectiveness was notably higher in patients with minimal or no residual disease. ${ }^{27}$

Remarkably, the combination of vaccine and checkpoint inhibition was uniquely capable of eradicating disease and producing long-term survival in all of the animals. In addition, animals treated with the combination of vaccination and checkpoint inhibition were protected from leukemia engraftment after re-challenge with an otherwise lethal dose of leukemia cells, an effect consistent with generation of a memory response with long-term efficacy. The therapeutic efficacy of vaccination and combined checkpoint blockade was largely reproduced by vaccination with PD-1 blockade alone, whereas vaccination with TIM3 blockade alone enhanced the initial response to the vaccine but resulted in only a subset of animals being resistant to rechallenge with the tumor. Interestingly, vaccination in combination with anti-RGMb did not affect the animals' survival in this model. This observation could in part be explained by the lack of PD-L2 expression by TIB-49 AML tumor cells (data not shown), which would prevent signaling through that inhibitory pathway.

To further elucidate the immunological mechanism responsible for these observations, we studied the impact of therapy on leukemia-specific T-cell immunity in each of the differently treated cohorts of animals. Consistent with the underlying hypothesis, checkpoint inhibition did not increase the relative prevalence of leukemia-specific $T$ cells above that present in untreated animals challenged with tumor cells. Of note, while vaccination with DC/AML fusions resulted in the expansion of leukemia-specific $T$ cells in the peripheral blood and spleen, the levels of these cells were nearly doubled when animals were treated with the combination of vaccination and checkpoint inhibition. This demonstrated an important concept that while checkpoint blockade was not able to induce primary leukemiaspecific immunity as a single modality, it was capable of further expanding vaccine-educated $\mathrm{T}$ cells. These findings were reproduced in an additional immunocompetent murine model of primary inducible IDH2 leukemia. Mice treated with a combination of a syngeneic fusion vaccine and checkpoint blockade demonstrated significantly decreased tumor burden as well as a 4-fold expansion of AML specific T cells.

Moreover, assessment of antigen-specific antitumor immunity revealed a significant increase in the frequency of survivin-specific $T$ cells, as demonstrated by an increase in $\mathrm{T}$ cells positive for CD8 and survivin pentamers following vaccination and checkpoint inhibition compared to the numbers of these cells in controls. Interestingly, no change was observed in the frequency of cytomegalovirus-specific $\mathrm{T}$ cells, suggesting the tumor-specific nature of the T-cell activation and expansion.

The nature of the immune response following vaccination and checkpoint blockade was further interrogated by single-cell RNA sequencing, which demonstrated comparatively increased activation of signaling pathways regulating cell viability, proliferation and survival among effector and memory $T$ cells, and downregulation of apoptosis-regulating genes. Functional memory $\mathrm{T}$ cells have been shown to be a critical immune subset, with their presence being predictive of more durable responses following immunotherapy. ${ }^{28}$ Furthermore, signaling pathway analysis within CD8 and CD4 memory T-cell compartments demonstrated significant upregulation of crucial pathways such as CD28 and TCR signaling, as well as mTOR signaling, known to be important in memory T-cell differentiation, ${ }^{29}$ following vaccination as compared to the state in untreated controls. Interestingly, this effect was enhanced, with further upregulation, following the addition of checkpoint blockade. Analysis of signaling in effector CD8/Nkg7 T cells showed significant upregulation of NF- $\kappa \mathrm{B}, \mathrm{mTOR}, \mathrm{CD} 28$ and ICOS after vaccination, consistent with induction of an inflamma-

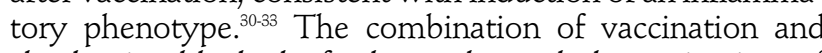
checkpoint blockade further enhanced the activation of these signaling pathways.

In the context of enhanced expansion of tumor reactive lymphocytes and activation of TCR signaling pathways, we subsequently quantified the effect of vaccination in combination with checkpoint blockade on T-cell diversity repertoire. Vaccination with DC/AML fusions resulted in enhanced clonal diversity and the oligoclonal expansion of specific TCR sequences, consistent with vaccine-mediated targeting of tumor-associated epitopes. Importantly the addition of checkpoint inhibitors further enhanced this 
response, promoting clonal diversity, consistent with selective expansion of vaccine-educated clones. This finding supports the hypothesis that sequential expansion of vaccine-educated lymphocytes followed by checkpoint blockade would selectively promote vaccine-mediated tumor-specific immune responses. It has been previously demonstrated that antigen-specific activation of $\mathrm{T}$ cells is associated with upregulation of PD-1 expression following chemotherapy among patients treated for AML, ${ }^{34}$ potentially offering a selectively timed target for PD-1 blockade. Based on this premise, checkpoint blockade in the context of vaccination may amplify the tumor-specific response in preference to nonspecific activation of autoreactive clones. While a similar amplification of vaccineinduced autoimmunity is possible, there was no significant evidence of this in the vaccinated patients or the animals treated with the combination. We have now initiated a clinical trial of $\mathrm{DC} /$ tumor vaccination in combination with PD-1 blockade in which therapeutic efficacy and toxicity will be assessed.

\section{Disclosures}

$J R$ has received research support from Celgene and BMS; sat on advisory boards for Amgen, Merck, BMS and Partner Tx; acted as a consultant for Parexel and Imaging Endpoint; and provided educational services for Dava Oncology. DA has received research support from Celgene and Pharmacyclics; has sat on advisory boards for Celgene, Juno, Partners Tx, Karyopharm, BMS and Aviv MedTech Ltd; and acted as a consultant for Janssen, Parexel and Takeda. GJF has patents/pending royalties on the PD-1/PDL1 pathway from Roche, Merck, BMS, EMD-Serono, Boehringer-Ingelheim, AstraZeneca, Dako and Novartis; patents on the TIM-3 and RGMb pathways licensed to Novartis; equity in Nextpoint and Triursus; and served on advisory boards for Roche, BMS, Xios and Origime

\section{Contributions}

DS and JL designed and performed research, interpreted data, and were responsible for preparing the manuscript. $M B$ designed and directed research including studies related to TCR clonotype and single-cell analysis. SB performed SCRNA-sequencing experiments and analysis. BT performed scRNA-sequencing and targeted TCR sequencing. GC performed the $\mathrm{IIDH} 2$ in vivo experiments. RP analyzed TCR sequencing data. KM, MN, SO, MC, $M W, S J, L B, H G, A M, B T, D T$ and $C T$ performed research studies and participated in designing the research. BE, $R S, D K$ and GF assisted with research design, analyzed and interpreted data, and assisted with the manuscript preparation. DA and JR designed and supervised research, the associated analysis, and manuscript preparation.

\section{Acknowledgments}

We thank the animal core facility at Beth Israel Deaconess Medical Center for assistance with imaging and treatment. We also thank Prof. Irmela Jeremias from HelmholtzZentrum München, German Research Center for Environmental Health, for generously providing the $m$ Cherry/luciferase vector.

\section{Funding}

Research reported in this publication was supported in part by the National Cancer Institute of the National Institutes of Health under award \#P50CA206963. The content is solely the responsibility of the authors and does not necessarily represent the official views of the National Institutes of Health.

\section{References}

1. Ding L, Ley TJ, Larson DE, et al. Clonal evolution in relapsed acute myeloid leukaemia revealed by whole-genome sequencing. Nature. 2012;481(7382):506-510.

2. Champlin R. Reduced intensity allogeneic hematopoietic transplantation is an established standard of care for treatment of older patients with acute myeloid leukemia. Best Pract Res Clin Haematol. 2013;26(3):297300.

3. Petersen SL. Alloreactivity as therapeutic principle in the treatment of hematologic malignancies. Studies of clinical and immunologic aspects of allogeneic hematopoietic cell transplantation with nonmyeloablative conditioning. Dan Med Bull. 2007;54(2):112-139.

4. Rosenblatt J, Avivi I, Vasir B, et al. Vaccination with dendritic cell/tumor fusions following autologous stem cell transplant induces immunologic and clinical responses in multiple myeloma patients. Clin Cancer Res. 2013;19(13):3640-3648.

5. Rosenblatt J, Vasir B, Uhl L, et al. Vaccination with dendritic cell/tumor fusion cells results in cellular and humoral antitumor immune responses in patients with multiple myeloma. Blood. 2011:117(2):393-402.

6. Rosenblatt J, Stone RM, Uhl L, et al. Individualized vaccination of AML patients in remission is associated with induction of antileukemia immunity and prolonged remissions. Sci Transl Med. 2016;8(368): 368ra171.

7. Almeida AM, Ramos F. Acute myeloid leukemia in the older adults. Leuk Res Rep. 2016;6:1-7.

8. Zarour HM. Reversing T-cell dysfunction and exhaustion in cancer. Clin Cancer Res. 2016;22(8):1856-1864.

9. Łuksza M, Riaz N, Makarov V, et al. A neoantigen fitness model predicts tumour response to checkpoint blockade immunotherapy. Nature. 2017;551(7681): 517-520.

10. Daver N, Garcia-Manero G, Basu S, et al. Efficacy, safety, and biomarkers of response to azacitidine and nivolumab in relapsed/refractory acute myeloid leukemia: a nonrandomized, open-label, phase II study. Cancer Discov. 2019;9(3):370-383.

11. Mahoney KM, Rennert PD, Freeman GJ. Combination cancer immunotherapy and new immunomodulatory targets. Nat Rev Drug Discov. 2015;14(8):561-584.

12.Zhou $\mathrm{Q}$, Munger ME, Veenstra RG, et al. Coexpression of Tim-3 and PD-1 identifies a CD8+ T-cell exhaustion phenotype in mice with disseminated acute myelogenous leukemia. Blood. 2011;117(17):4501-4510.

13. Sakuishi K, Apetoh L, Sullivan JM, Blazar BR, Kuchroo VK, Anderson AC. Targeting Tim-3 and PD-1 pathways to reverse T cell exhaustion and restore anti-tumor immunity. J Exp Med. 2010;207(10):2187-2194.

14. Kikushige Y, Miyamoto T, Yuda J, et al. A TIM-3/Gal-9 autocrine stimulatory loop drives self-renewal of human myeloid leukemia stem cells and leukemic progression. Cell Stem Cell. 2015;17(3):341-352.

15. Severyn CJ, Shinde U, Rotwein P. Molecular biology, genetics and biochemistry of the repulsive guidance molecule family. Biochem J. 2009;422(3):393-403.

16. Raymond A, Liu B, Liang $\mathrm{H}$, et al. A role for BMP-induced homeobox gene MIXL1 in acute myelogenous leukemia and identification of type I BMP receptor as a potential target for therapy. Oncotarget. 2014;5(24): 12675-12693.

17. Xiao Y, Yu S, Zhu B, et al. RGMb is a novel binding partner for PD-L2 and its engagement with PD-L2 promotes respiratory tolerance. J Exp Med. 2014;211(5):943-959

18. Mugoni V, Panella R, Cheloni G, et al. Vulnerabilities in mIDH2 AML confer sensitivity to APL-like targeted combination therapy. Cell Res. 2019;29(6):446-459.

19. Nahas MR, Stroopinsky D, Rosenblatt J, et al. Hypomethylating agent alters the immune microenvironment in acute myeloid leukaemia (AML) and enhances the immunogenicity of a dendritic cell/AML vaccine. Br J Haematol. 2019;185(4):679-690

20. Giudicelli V, Chaume D, Lefranc MP IMGT/GENE-DB: a comprehensive database for human and mouse immunoglobulin and T cell receptor genes. Nucleic Acids Res. 2005;33(Database issue):D256-261.

21. Bolotin DA, Poslavsky S, Mitrophanov I, et al. MiXCR: software for comprehensive adaptive immunity profiling. Nat Methods. 2015;12(5):380-381.

22. Butler A, Hoffman P, Smibert P, Papalexi E, Satija R. Integrating single-cell transcriptomic data across different conditions, technologies, and species. Nat Biotechnol. 2018;36 (5):411-420

23. Becht E, McInnes L, Healy J, et al. 
Dimensionality reduction for visualizing single-cell data using UMAP. Nat Biotechnol. 2018 Dec 3. doi: 10.1038/nbt.4314. [Online ahead of print].

24. Satija R, Farrell JA, Gennert D, et al. Spatial reconstruction of single-cell gene expression data. Nat Biotechnol. 2015;33(5):495-502.

25. Brahmer JR, Tykodi SS, Chow LOM, et al. Safety and activity of anti-PD-L1 antibody in patients with advanced cancer. N Engl J Med. 2012;366(26):2455-2465.

26. Stahl M, Goldberg AD. Immune checkpoint inhibitors in acute myeloid leukemia: novel combinations and therapeutic targets. Curr Oncol Rep. 2019;21(4):37.

27. Hale DF, Vreeland TJ, Peoples GE. Arming the immune system through vaccination to prevent cancer recurrence. Am Soc Clin Oncol Educ Book. 2016;(36):e159-e167.

28. Zuazo M, Arasanz H, Fernández-Hinojal G, et al. Functional systemic CD4 immunity is required for clinical responses to $\mathrm{PD}-\mathrm{L} 1 / \mathrm{PD}$ 1 blockade therapy. EMBO Mol Med. 2019;11(7):e10293.

29. Araki K, Turner AP, Shaffer VO, et al. mTOR regulates memory CD8 T-cell differentiation. Nature. 2009;460(7251):108-112.

30. Liu T, Zhang L, Joo D, Sun S-C. NF-кB signaling in inflammation. Signal Transduct Target Ther. 2017;2:17023.

31. Peter C, Waldmann H, Cobbold SP. mTOR signalling and metabolic regulation of $\mathrm{T}$ cell differentiation. Curr Opin Immunol. 2010 22(5):655-661

32. Thompson CB, Lindsten T, Ledbetter JA, et al. CD28 activation pathway regulates the production of multiple T-cell-derived lymphokines/cytokines. Proc Natl Acad Sci U S A. 1989;86(4):1333-1337.

33. Dong C, Juedes AE, Temann UA, et al. ICOS co-stimulatory receptor is essential for T-cell activation and function. Nature. 2001:409 (6816):97-101

34. Sehgal A, Whiteside TL, Boyiadzis M. Programmed death-1 checkpoint blockade in acute myeloid leukemia. Expert Opin Biol Ther. 2015;15(8):1191-1203 